\title{
Antibiotic treatment for uncomplicated and mild complicated diverticulitis: outpatient treatment for everyone
}

\author{
Gaëtan-Romain Joliat ${ }^{1}$. Jonathan Emery ${ }^{1}$ - Nicolas Demartines ${ }^{1} \cdot$ Martin Hübner $^{1}$. \\ Bertrand Yersin $^{2}$ • Dieter Hahnloser ${ }^{1}$
}

Accepted: 20 June 2017 / Published online: 29 June 2017

(C) Springer-Verlag GmbH Germany 2017

\begin{abstract}
Purpose Antibiotic treatment is the treatment of choice for uncomplicated diverticulitis (uD) and can be performed for mild complicated diverticulitis (mcD). In several cases, outpatient treatment (OT) can be undertaken. This study assessed the 1-month failure rate of OT for $\mathrm{uD} / \mathrm{mcD}$ compared to inpatient treatment (IT), and identified predictive factors for treatment failure.

Methods All consecutive patients (2006-2012) diagnosed with $\mathrm{uD} / \mathrm{mcD}$ by $\mathrm{CT}$ scan were retrospectively analyzed. Acute $\mathrm{uD}$ was defined as absence of the following: abscess, fistula, extraluminal contrast, pneumoperitoneum, and need for immediate percutaneous drainage/surgery. Acute $\mathrm{mcD}$ was defined as complicated diverticulitis with abscess $<4 \mathrm{~cm}$ or pneumoperitoneum $<2 \mathrm{~cm}$. All patients received antibiotherapy. Treatment failure was defined as (re)hospitalization the first month after treatment onset or need of drainage/surgery during hospitalization. All patients were contacted using a standardized questionnaire. Results Out of $540 \mathrm{uD} / \mathrm{mcD}$, IT was offered to 369 patients $(68 \%)$ and OT to 171 patients (32\%). The IT group had higher median age, more women, higher median Charlson Index, more severe median Ambrosetti score, longer median time in the emergency room, and higher median CRP. Response
\end{abstract}

This paper has been presented in parts at the 102nd Annual Congress of the Swiss Surgical Society, May 20-22, 2015, Bern, Switzerland.

Nicolas Demartines

demartines@chuv.ch

1 Department of Visceral Surgery, Lausanne University Hospital (CHUV), Rue du Bugnon 46, 1011 Lausanne, Switzerland

2 Emergency Department, Lausanne University Hospital (CHUV), Lausanne, Switzerland rates to the questionnaire were $56 \%$ (IT) vs. $62 \%$ (OT), $p=0.18$. Failure rates were $32 \%$ in IT vs. $10 \%$ in OT group, $p<0.01$. Among the $\mathrm{uD} / \mathrm{mcD}$ patients, admission/CT time between midnight and 6 AM, Ambrosetti score of 4, and free air around the colon were risk factors for failure.

Conclusions Outpatient treatment for uncomplicated/mild complicated diverticulitis is feasible and safe. Prognostic factors of failure necessitating closer follow-up were admission/ CT time, Ambrosetti score of 4, and free air around the colon.

Keywords Diverticulitis · Outpatient treatment $\cdot$ Treatment failure $\cdot$ Antibiotics

\section{Introduction}

Approximately $20 \%$ of patients with diverticular disease will present at least one episode of acute diverticulitis [1-4]. Recently, the treatment of diverticulitis has evolved to become more conservative even without antibiotics [5] and less invasive with percutaneous drainage or laparoscopic lavage [6]. Indications for emergency surgery are now restricted to gross purulent or fecal peritoneal contamination, septic shock, or failure of conservative treatment [7]. In addition, the majority of patients present with uncomplicated diverticulitis (uD), i.e., without complications such as abscess, bleeding, fistula, stenosis, or perforation with free air [8]. Patients with small abscess or low free air (mild complicated diverticulitis, $\mathrm{mcD}$ ) can also be treated with antibiotics according to recent studies [8]. Several studies showed that outpatient treatment (OT) of $\mathrm{uD}$ and $\mathrm{mcD}$ was feasible and safe [9-11]. It has also been shown that OT was cost-beneficial and costeffective inducing important savings for the hospital and the general health care system $[10,12,13]$. 
However, decision for OT with antibiotherapy is often made subjectively by the doctor and depends on various not well-defined factors such as general health condition of the patient, ability to tolerate oral medication (no nausea or vomiting), absence of diarrhea, or support at home. For daily clinical practice, only few studies have investigated predictive factors of OT failure for $\mathrm{uD}[9,14]$.

The aim of the present study was to assess the 1-month failure rate of antibiotic $\mathrm{OT}$ for $\mathrm{uD}$ and $\mathrm{mcD}$ compared to antibiotic inpatient treatment (IT), and to define predictive factors of treatment failure.

\section{Material and methods}

\section{Patients, inclusion and exclusion criteria}

All patients with $\mathrm{uD}$ and $\mathrm{mcD}$ presenting to the emergency room (ER) of the Lausanne University Hospital (CHUV) from January 2006 to December 2012 were retrospectively analyzed. Inclusion criteria were patients $>18$ years old and CTbased diagnosis of $\mathrm{uD}$, i.e., diverticulitis without complication, or $\mathrm{mcD}$. Complications of acute diverticulitis were defined in our study as extraluminal extravasation of contrast product on CT, pneumoperitoneum, presence of fistula, intra-abdominal or pericolic abscess, bleeding, or stenosis. Acute $\mathrm{mcD}$ was defined as patients with complicated diverticulitis with pneumoperitoneum $<2 \mathrm{~cm}$ under the diaphragm or intra-abdominal/pericolic abscess $<4 \mathrm{~cm}$. Patients requiring an immediate (at admission) percutaneous drainage or surgery were excluded from the analysis. The number of prior diverticulitis episodes was not an exclusion criterion.

\section{Outcome measures and questionnaire}

Patients' characteristics, symptoms, laboratory tests, treatment data, and orientation after diagnosis (home, hospital, transfer to another hospital) were recorded and retrospectively reviewed. Comorbidities of the patients were scored using the Charlson Comorbidity Index [15]. Severity of uD and $\mathrm{mcD}$ was graded by the CT scan-based classification of Ambrosetti, where diverticulitis was subdivided into moderate $(1=$ localized colic wall thickening $>5 \mathrm{~mm}, 2=$ pericolic fat infiltration) and severe ( $3=$ abscess, $4=$ extraluminal air or contrast) [16]. Treatment failure was defined as reappearance of symptoms and (re)hospitalization during the month following the start of antibiotic treatment, or need of drainage/ surgery during primary hospitalization (inpatient group). Recurrence was defined as new symptoms appearing more than 1 month after initial treatment. All included patients were contacted using a standardized questionnaire to assess treatment failure. The questionnaires to the patients were sent in 2014. In case of non response, patients were sent the questionnaire one more time. The questionnaire asked if the patient had to be hospitalized for diverticulitis the month following the initial treatment, if he had to be operated during that hospitalization, if he had new symptoms more than 1 month after treatment, if he had a control colonoscopy (recommended for all patients $>50$ years of age and if $>5$ years from last colonoscopy), and if he underwent elective colon surgery during follow-up. Incomplete or unclear returned questionnaires were completed by contacting the general practitioner and/or the patient when needed.

\section{Outpatient and inpatient treatments}

All patients with $\mathrm{uD}$ and $\mathrm{mcD}$ received antibiotics independently of the treatment type (OT or IT).

All cases of diverticulitis diagnosed in the ER were presented to the surgeon on call or to the responsible ER senior physician. The decision of undertaking an OT was decided case by case by the surgeon or the ER physician. The elements motivating the decision were the patient's ability to tolerate oral medication, pain control with oral medications, and presence of support at home. In case of OT, one single shot of intravenous (IV) antibiotics was given in the ER, and then a course of oral antibiotics was ordered for a period of 10 days based on our institutional guidelines.

If the patient was hospitalized, IV antibiotics and IV fluids were started immediately. Antibiotics were switched to oral form when the pain was controlled by non-opioid analgesics (e.g., paracetamol or metamizole) and when the patient was able to tolerate oral medication. There was no alimentary restriction during the hospitalization. Patients were discharged once antibiotics were given orally and pain controlled with oral analgesics.

\section{Statistical analysis}

Comparisons between groups for continuous variables were done by using a Mann-Whitney $U$ test or a $t$ test depending on the variable distribution and the variance homogeneity. Fisher's exact test was used for discrete variables. GraphPad Prism@ 5 for Mac OS X (GraphPad Software Inc.) was used for calculation and analysis. A $p$ value $<0.05$ was considered to be statistically significant.

The study protocol was granted approval by the local Ethics Committee (protocol number: 255/13100613).

\section{Results}

\section{Patient demographics and treatment characteristics}

During the study period, 540 patients with $\mathrm{uD}$ and $\mathrm{mcD}$ were collected (Fig. 1). Among these 540 patients, 68\% (369) 
Fig. 1 Flowchart of the study patients

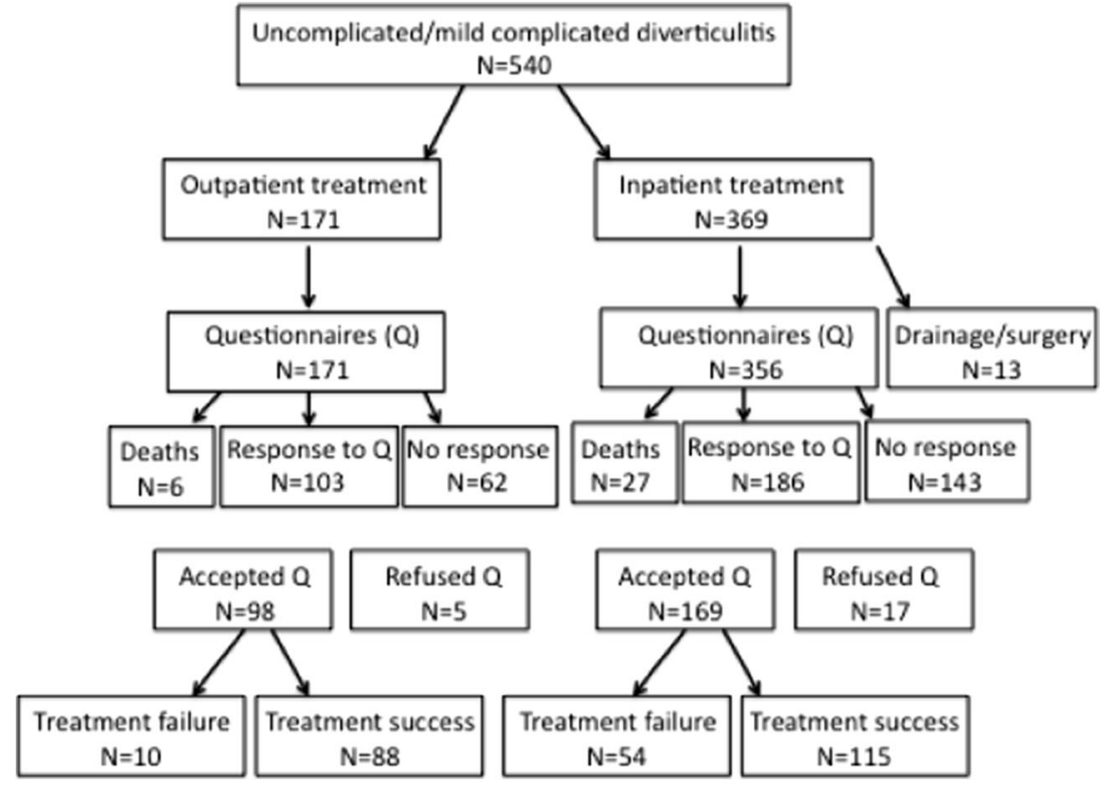

underwent IT and 32\% (171) OT. For the IT group, the median length of stay was 4 days (IQR, 3-5). The number of first $\mathrm{uD}$ and $\mathrm{mcD}$ episodes was $263(71 \%)$ and $123(72 \%)$ in the IT and OT groups, respectively $(p=0.92)$. The percentage of OT increased over time (2006: $12 / 92=13 \%, 2012: 40 /$ $104=38 \%, p<0.001)$. Overall, the IT group had a higher median age compared to the OT group $(p<0.001)$, comprised more women $(p=0.003)$, had a higher median Charlson Index $(p<0.001)$, comprised a higher number of high Ambrosetti scores $(p<0.001)$, had a longer median stay in the ER $(p<0.001)$, and had a higher median CRP $(p<0.001)$. Demographics were otherwise similar between the two groups (Table 1). Moreover, the two groups of patients treated from 2006 to 2008 and from 2009 to 2012 (corresponding to half of the study period) had no statistical difference in demographics and disease presentation, in parallel to the increased percentage of OT over the years.

\section{Treatment outcomes}

Twenty-seven patients in the IT group and 6 in the OT group were dead (unrelated to diverticulitis) at the time of questionnaire sending in 2014. The response rates of alive patients to the questionnaires were $56 \%(186 / 329)$ in the IT and $62 \%$ $(103 / 165)$ in the OT $(p=0.25)$. There were no demographic differences between responders and non-responders (data not shown). Among the patients who responded and accepted the study (Qgroup: 267 patients, 22 refusals), 32\% (54/169) mentioned a hospitalization for the same abdominal pain during the month after treatment in the IT group compared to $10 \%$ $(10 / 98)$ in the OT group $(p<0.001)$. Between these latter two groups, there were no statistical differences in demographics, laboratory results, episode number, and antibiotherapy length.
Among the Qgroup, 30/169 (18\%) had an elective operation in the IT group and $14 / 98(13 \%)$ in the OT group $(p=0.50)$. The median follow-up in the Qgroup was 59.5 and 46.5 months for the IT and OT groups, respectively $(p=0.04)$. Recurrences during the follow-up (new symptoms appearing more than 1 month after initial treatment) were 40 / $98(41 \%)$ in the OT group and 70/169 (41\%) in the IT group $(p=1)$.

\section{Treatment failure}

Treatment failure necessitating drainage or surgery during the hospitalization (not at admission, but during primary stay due to deterioration) occurred in 13 patients ( $4 \% ; 5$ women; 8 operations and 5 percutaneous drainages) in the IT group. There were no significant demographic differences between these 13 patients and the 356 other patients who had an IT (data not shown). Extraluminal air was associated with higher rate of IT failure $(p=0.02)$, and $\mathrm{uD} / \mathrm{mcD}$ episode $>2$ was associated with higher rate of OT failure $(p=0.02)$. Colonoscopy between 4 and 12 weeks after the acute episode was performed in 199 patients (70 in the OT group, 129 in the IT group). The results of IT and OT are summarized in Table 2.

\section{Risk factors for treatment failure}

There was no difference in gender $(p=0.89)$ and age $(p=0.89)$ between failures and successful treatments. There were more treatment failures when admission time and CT time were between midnight and $6 \mathrm{AM}(p=0.008$ and $p=0.007$, respectively), but no difference regarding the trimester (season) of admission date. There was also no difference between the two groups concerning the time spent in the 
Table 1 Patient demographics in the outpatient treatment group (OT) and in the inpatient treatment group (IT)

\begin{tabular}{|c|c|c|c|}
\hline & $\begin{array}{l}\text { OT } \\
n=171\end{array}$ & $\begin{array}{l}\text { IT } \\
n=369\end{array}$ & $p$ value \\
\hline Age (years) & $53(44-64)$ & $61(50-72)$ & $<0.001$ \\
\hline Women/men & $62 / 109$ & $185 / 184$ & 0.003 \\
\hline Charlson Index [15] & $2(1-3)$ & $3(2-4)$ & $<0.001$ \\
\hline Ambrosetti stage $1 / 2 / 3 / 4$ [16] & $9 / 149 / 7 / 6$ & $9 / 250 / 43 / 67$ & $<0.001$ \\
\hline Number of 1 st episode & $123(72 \%)$ & $263(71 \%)$ & 0.918 \\
\hline Right colon & $6(4 \%)$ & $25(7 \%)$ & 0.164 \\
\hline Alive/dead & $165 / 6$ & $342 / 27$ & 0.086 \\
\hline Time spent in the $\mathrm{ER}^{\mathrm{a}}(\mathrm{min})$ & $310(237-396)$ & $345(273-437)$ & $<0.001$ \\
\hline Time between admission and CT (min) & $173(113-256)$ & $171(121-246)$ & 0.935 \\
\hline Symptom duration (h) & $48(24-72)$ & $48(24-96)$ & 0.615 \\
\hline Previous abdominal surgery & $85(50 \%)$ & $213(58 \%)$ & 0.080 \\
\hline Mean arterial pressure $(\mathrm{mmHg})^{\mathrm{b}}$ & $99(89-108)$ & $100(89-108)$ & 0.747 \\
\hline Heart rate $^{\mathrm{b}}$ & $84(74-92)$ & $84(73-93)$ & 0.942 \\
\hline Temperature $\left({ }^{\circ} \mathrm{C}\right)^{\mathrm{b}}$ & $36.7(36.2-37.2)$ & $36.9(36.5-37.5)$ & 0.242 \\
\hline Leukocyte count $(\mathrm{G} / \mathrm{l})^{\mathrm{b}}$ & $11(8-13)$ & $11(9-13)$ & 0.185 \\
\hline C-reactive protein level $\left(\mathrm{mg} / \mathrm{l}^{\mathrm{b}}\right.$ & $42(20-73)$ & $77(37-133)$ & $<0.001$ \\
\hline Thrombocyte level $(\mathrm{G} / \mathrm{l})^{\mathrm{b}}$ & $233(202-274)$ & $236(197-275)$ & 0.967 \\
\hline Creatinine level $(\mu \mathrm{g} / \mathrm{l})^{\mathrm{b}}$ & $80(68-89)$ & $81(69.5-96.5)$ & 0.060 \\
\hline
\end{tabular}

All values are median (interquartile range) or number (\%). Significant $p$ values appear in italics.

${ }^{\mathrm{a}}$ Emergency room

${ }^{\mathrm{b}}$ At admission

ER $(p=0.91)$. The median Charlson Index was similar. There were significantly more Ambrosetti grade 2 and fewer grade 4 in the group with no treatment failure. Free air around the colon was associated with a statistically significantly increased risk of failure $(p=0.0004)$ (Table 3).

\section{Discussion}

The present study showed that antibiotic OT of acute uD and $\mathrm{mcD}$ was feasible and safe with a low 1-month failure rate of $10 \%$ compared to $32 \%$ for antibiotic IT. Predictive factors of treatment failure for OT and IT were an ER admission time and a $\mathrm{CT}$ performed between midnight and $6 \mathrm{AM}$, an Ambrosetti score of 4, and the presence of free air around the colon.
The reason why an admission time and a CT between midnight and $6 \mathrm{AM}$ were associated with an increased risk of treatment failure is not evident. It might be that patients are more reluctant to come to the ER during night meaning that those coming at night have a more severe disease. However, we included only $\mathrm{uD}$ and $\mathrm{mcD}$, and all $\mathrm{CT}$ scans were reviewed by a senior radiologist in the morning. If the diverticulitis was not uncomplicated or mild complicated as defined above according to our criteria, patients were excluded from the study. It is also possible that a circadian variation can be implicated or that this finding resulted from an out-of-hour effect. Etzioni et al. found in a retrospective study that being a woman and having free fluid on the CT were risk factors for OT failure [9]. Similarly to the results of this study, age, laboratory results, or the Charlson Index were not prognostic factors of failure in our cohort. Of note, in their study
Table 2 Outcomes of responders $(n=267)$ by treatment group

\begin{tabular}{llll}
\hline & $\begin{array}{l}\text { OT } \\
n=98\end{array}$ & $\begin{array}{l}\text { IT } \\
n=169\end{array}$ & $p$ value \\
\hline Median follow-up (months) & $46.5(29-74)$ & $59.5(34-82)$ & 0.035 \\
Treatment failure & $10(10 \%)$ & $54(32 \%)$ & $<0.001$ \\
If treatment failure, operation during hospital stay & $3(3 \%)$ & $13(8 \%)$ & 0.701 \\
Elective colic resection & $14(13 \%)$ & $30(18 \%)$ & 0.497 \\
Colonoscopy after episode & $70(71 \%)$ & $129(76 \%)$ & 0.380 \\
\hline
\end{tabular}

Significant $p$ values appear in italics. 
Table 3 Characteristics of patients with treatment failure and with no treatment failure

\begin{tabular}{|c|c|c|c|}
\hline & $\begin{array}{l}\text { Treatment failure } \\
n=64\end{array}$ & $\begin{array}{l}\text { No treatment failure } \\
n=203\end{array}$ & $p$ value \\
\hline Women/men & $31 / 33$ & $96 / 107$ & 0.887 \\
\hline Age (years) & $60(48.5-69.5)$ & $58.9(50.4-69)$ & 0.887 \\
\hline \multicolumn{4}{|l|}{ Admission time } \\
\hline 00:00-6:00 & 13 & 11 & 0.008 \\
\hline $6: 01-12: 00$ & 21 & 78 & 0.460 \\
\hline $12: 01-18: 00$ & 16 & 65 & 0.350 \\
\hline $18: 01-23: 59$ & 14 & 49 & 0.866 \\
\hline \multicolumn{4}{|l|}{ Admission date } \\
\hline $1 / 1-31 / 3$ & 12 & 31 & 0.559 \\
\hline $1 / 4-30 / 6$ & 17 & 58 & 0.874 \\
\hline $1 / 7-30 / 9$ & 24 & 62 & 0.357 \\
\hline $1 / 10-31 / 12$ & 11 & 52 & 0.181 \\
\hline \multicolumn{4}{|l|}{ CT time ${ }^{\mathrm{a}}$} \\
\hline 00:00-6:00 & 14 & 18 & 0.007 \\
\hline $6: 01-12: 00$ & 10 & 29 & 0.686 \\
\hline $12: 01-18: 00$ & 24 & 92 & 0.378 \\
\hline $18: 01-23: 59$ & 12 & 58 & 0.186 \\
\hline Time spent in the $\mathrm{ER}^{\mathrm{b}}(\mathrm{min})$ & $164.5(113.8-233)$ & $181.5(99-254.8)$ & 0.906 \\
\hline Charlson Index [15] & $3(1-4)$ & $3(2-4)$ & 0.869 \\
\hline Episode number $>2$ & $55(86 \%)$ & $190(94 \%)$ & 0.067 \\
\hline Ambrosetti score [16] & & & 0.001 \\
\hline 1 & 3 & 6 & 0.451 \\
\hline 2 & 38 & 163 & 0.001 \\
\hline 3 & 6 & 17 & 0.801 \\
\hline 4 & 17 & 17 & 0.0004 \\
\hline \multicolumn{4}{|l|}{ Free air on $\mathrm{CT}$ : } \\
\hline - In the pericolic fat & 8 & 15 & 1 \\
\hline - Around the colon & 12 & 8 & 0.0004 \\
\hline Symptom length (hours) & $48(16.5-72)$ & $48(24-72)$ & 0.153 \\
\hline Right/left colon & $3 / 61$ & 9/194 & 1 \\
\hline Mean arterial pressure $(\mathrm{mmHg})^{\mathrm{c}}$ & $97(88-112)$ & $100(89-108)$ & 0.752 \\
\hline Temperature $\left({ }^{\circ} \mathrm{C}\right)^{\mathrm{c}}$ & $36.8(36.5-37.5)$ & $36.8(36.4-37.3)$ & 0.321 \\
\hline Heart rate ${ }^{c}$ & $81(70-90)$ & $84(74-92)$ & 0.284 \\
\hline CRP level (mg/lic & $54(29-113)$ & $63(32-116)$ & 0.565 \\
\hline Leukocyte count $(\mathrm{G} / \mathrm{l})^{\mathrm{c}}$ & $11.3(9.1-13.5)$ & $11.5(9.5-13.5)$ & 0.794 \\
\hline Thrombocyte level $(\mathrm{G} / \mathrm{l})^{\mathrm{c}}$ & $238(194-277)$ & $237(198-269)$ & 0.856 \\
\hline Creatinine level $(\mu \mathrm{g} / \mathrm{l})^{\mathrm{c}}$ & $77(67-87)$ & $80(69-92)$ & 0.233 \\
\hline
\end{tabular}

All values are median (interquartile range) or number (\%). Significant $p$ values appear in italics.

${ }^{\text {a }} \mathrm{CT}$ times were missing for 10 patients (4 in the failure group and 6 in the non failure group)

${ }^{\mathrm{b}}$ Emergency room

${ }^{\mathrm{c}}$ At admission

Etzioni et al. found a failure rate of $6 \%$, in line with the $10 \%$ of failure that we observed in the OT group.

Patients with one of the above-mentioned risk factors for treatment failure should have a tighter outpatient follow-up. This follow-up can be undertaken with repetitive phone calls or with an early visit to their general practitioner [10]. Such institutional guidelines have been put in place since, and outcomes are now assessed prospectively.

For patients with $\mathrm{uD}$ and $\mathrm{mcD}$ who had IT, risk factors for early failure during the hospital stay could not be identified. However, early failure requiring drainage or surgery during the index hospitalization occurred in $4 \%$ of patients only. 
Despite the fact that no clear institutional guidelines for IT or OT were available during the study period, the percentage of OT for $\mathrm{uD}$ and $\mathrm{mcD}$ increased with time from 13 to $38 \%$. This observation corroborates the results of several articles that showed that OT was safe and feasible in case of $\mathrm{uD}$ and $\mathrm{mcD}[7,9-11,17]$. In a multicentric randomized controlled trial, Chabok et al. have even demonstrated that antibiotics were not superior to symptomatic treatment in terms of complications, recurrence, and recovery time [5]. Based on these data and the low failure rate, OT without antibiotics could be proposed in more patients. In the present study, all patients received antibiotherapy for $\mathrm{uD}$ and $\mathrm{mcD}$. Based on the results of the previously mentioned study [5] and the future results of an ongoing trial comparing antibiotics vs. no antibiotics [18], treatment is becoming more conservative. OT has several advantages. It allows decreasing the hospital costs normally induced by a hospitalization for $\mathrm{uD}$ and $\mathrm{mcD}$, and it can reduce the overload of hospitalized patients $[10,12]$. Another corollary of OT is the diminution of the risks of nosocomial infection [19]. Of note, our study did not assess the feasibility of OT without antibiotics as all patients received antibiotherapy in our protocol.

There is presently no clear consensus regarding the definition of $\mathrm{uD}$. In the current literature, abscess $<2$ or $<5 \mathrm{~cm}$ depending on the diverse guidelines is classified as $\mathrm{uD}$ or $\mathrm{mcD}$ $[7,8,20-22]$. Recently, the presence of free air $>2 \mathrm{~cm}$ or retroperitoneal air was shown to be risk factors for conservative treatment failure [14]. In the present study, an abscess $<4 \mathrm{~cm}$ was considered as $\mathrm{mcD}$ in light of recent literature. These patients were successfully treated as outpatients. However, the case number of this subgroup is too small for definitive recommendations.

Several limitations of this study need to be addressed. Firstly, due to its retrospective design it is possible that some patients or some data not mentioned in the charts were missed. Secondly, patients who were first treated by their general practitioner and then presented to the ER were also included. However, they were not considered as OT failure because their initial diagnosis was not based on CT scan. Thirdly, the survey could contain a potential recall bias. Finally, the outcomes of non-responders are not known. However, a response rate of 56 and $62 \%$ is not perfect but correct for an aged study population and is in line with the literature. Interpretations of the results should therefore be made with caution. Another strong point is that all diagnoses were based on systematic abdominal CT scans.

This study thus confirmed that OT is safe for $\mathrm{uD}$ and $\mathrm{mcD}$ with a low 1-month failure rate and a similar recurrence rate as IT. OT could be used more often without excess risk in all patients presenting with $\mathrm{uD}$ or $\mathrm{mcD}$. ER admission and CT time, severity of $\mathrm{uD} / \mathrm{mcD}$, and presence of free air were identified as risk factors for treatment failure in this study. The next step would be to develop a score based on the risk factors for treatment failure to guide the follow-up and to validate it with a prospective population.

Acknowledgements None.

\section{Compliance with ethical standards}

Conflict of interest The authors declare that they have no conflict of interest.

Ethical approval All procedures performed in studies involving human participants were in accordance with the ethical standards of the institutional research committee and with the 1964 Helsinki declaration and its later amendments or comparable ethical standards.

Informed consent Informed consent was obtained from all individual participants included in the study.

\section{References}

1. Sheth AA, Longo W, Floch MH (2008) Diverticular disease and diverticulitis. Am J Gastroenterol 103:1550-1556

2. Etzioni DA, Mack TM, Beart RW et al (2009) Diverticulitis in the United States: 1998-2005: changing patterns of disease and treatment. Ann Surg 249:210-217

3. Jacobs DO (2007) Clinical practice. Diverticulitis. N Engl J Med 57:2057-2066

4. Stollman N, Raskin JB (2004) Diverticular disease of the colon. Lancet 363:631-639

5. Chabok A, Påhlman L, Hjern F et al (2012) Randomized clinical trial of antibiotics in acute uncomplicated diverticulitis. Br J Surg 99:532-539

6. Jones OM, Stevenson ARL, Clark D et al (2008) Laparoscopic resection for diverticular disease: follow-up of 500 consecutive patients. Ann Surg 248:1092-1097

7. Vennix S, Morton DG, Hahnloser D et al (2014) Systematic review of evidence and consensus on diverticulitis: an analysis of national and international guidelines. Color Dis 16:866-878

8. Biondo S, Lopez Borao J, Millan M et al (2012) Current status of the treatment of acute colonic diverticulitis: a systematic review. Color Dis 14:e1-11

9. Etzioni DA, Chiu VY, Cannom RR et al (2010) Outpatient treatment of acute diverticulitis: rates and predictors of failure. Dis Colon Rectum 53:861-865

10. Biondo S, Golda T, Kreisler E et al (2014) Outpatient versus hospitalization management for uncomplicated diverticulitis: a prospective, multicenter randomized clinical trial (DIVER Trial). Ann Surg 259:38-44

11. Abbas MA, Cannom RR, Chiu VY et al (2013) Triage of patients with acute diverticulitis: are some inpatients candidates for outpatient treatment? Color Dis 15:451-457

12. Moya P, Arroyo A, Pérez-Legaz J et al (2012) Applicability, safety and efficiency of outpatient treatment in uncomplicated diverticulitis. Tech Coloproctol 16:301-307

13. Lorente L, Cots F, Alonso S et al (2013) Outpatient treatment of uncomplicated acute diverticulitis: impact on healthcare costs. Cir Esp 91:504-509

14. Sallinen VJ, Mentula PJ, Leppäniemi AK (2014) Nonoperative management of perforated diverticulitis with extraluminal air is safe and effective in selected patients. Dis Colon Rectum 57:875-881 
15. Charlson ME, Pompei P, Ales KL et al (1987) A new method of classifying prognostic comorbidity in longitudinal studies: development and validation. J Chronic Dis 40:373-383

16. Ambrosetti P, Jenny A, Becker C et al (2000) Acute left colonic diverticulitis - compared performance of computed tomography and water-soluble contrast enema: prospective evaluation of 420 patients. Dis Colon Rectum 43:1363-1367

17. Jackson JD, Hammond T (2014) Systematic review: outpatient management of acute uncomplicated diverticulitis. Int J Color Dis 29:775-781

18. Unlü C, de Korte N, Daniels L et al (2010) A multicenter randomized clinical trial investigating the cost-effectiveness of treatment strategies with or without antibiotics for uncomplicated acute diverticulitis (DIABOLO trial). BMC Surg 10:23

19. Rueda JC, Jimenez A, Caro A et al (2012) Home treatment of uncomplicated acute diverticulitis. Int Surg 97:203-209

20. Andeweg CS, Mulder IM, Felt-Bersma RJF et al (2013) Guidelines of diagnostics and treatment of acute left-sided colonic diverticulitis. Dig Surg 30:278-292

21. Rafferty J, Shellito P, Hyman NH et al (2006) Practice parameters for sigmoid diverticulitis. Dis Colon Rectum 49:939-944

22. Wong WD, Wexner SD, Lowry A et al (2000) Practice parameters for the treatment of sigmoid diverticulitis - supporting documentation. The Standards Task Force. The American Society of Colon and Rectal Surgeons. Dis Colon Rectum 43:290-297 